\title{
Establishment of a GIST-T1 gastrointestinal stromal tumour cell line resistant to imatinib mesylate
}

\author{
YONGJIAN ZHOU ${ }^{1 *}$, JIABI CHEN $^{2 *}$, XIAOYUAN WENG ${ }^{1}$, GUOSHENG LIN $^{1}$, \\ ZICHENG HUANG $^{3}$ and HANLI SHUI ${ }^{1}$ \\ ${ }^{1}$ Department of Gastric Surgery, Union Hospital of Fujian Medical University, Fuzhou, Fujian 350001; \\ ${ }^{2}$ Department of Urinary Surgery, The Second Hospital of Fujian Medical University; \\ ${ }^{3}$ Department of Digestive Medicine, Quanzhou First Hospital, Quanzhou, Fujian 362000, P.R. China
}

Received June 8, 2017; Accepted December 20, 2017

DOI: $10.3892 / \mathrm{ol} .2018 .8283$

\begin{abstract}
In the present study, imatinib mesylate (IM) was used to induce resistance in the gastrointestinal stromal tumour (GIST) cell line, GIST-T1, to establish a stable resistant cell line. The growth characteristics and expression profile of the established cell line were compared with those of the parental cell line. Additionally, the resistance mechanism of the gastrointestinal stromal tumours was preliminarily investigated. The GIST-T1 cells were cultured in vitro, and the drug was administered in the logarithmic phase of cell growth using intermittent dosing with increasing concentrations to obtain a drug-resistant cell line by repeated induction. Differences in the biological behaviours of the parental cells and drug-resistant cells were examined, and changes in the expression profiles were compared in the two cell lines. The results showed that the IM-resistant GIST-T1 cell line (GIST-T1 IR) was successfully established. Analysis of the biological behaviours of the two cell lines revealed that the average doubling times of the parental cells and drug-resistant cells were 26.59 and $33.63 \mathrm{~h}$, respectively. The results of a scratch migration assay revealed that the migration ability was enhanced in the GIST-T1 IR cells. The results of CCK-8 detection indicated that the half maximal inhibitory concentration values of the two types of cells were 10.5 and $42.0 \mu \mathrm{M}$, respectively, which represented an increase of $\sim 4$-fold in the GIST-T1 IR cells.
\end{abstract}

Correspondence to: Dr Zicheng Huang, Department of Digestive Medicine, Quanzhou First Hospital, 248-252 East Street, Quanzhou, Fujian 362000, P.R. China

E-mail: hzcpwm@126.com

Dr Hanli Shui, Department of Gastric Surgery, Union Hospital of Fujian Medical University, 29 Xinquan Road, Fuzhou, Fujian 350001, P.R. China

E-mail: 149471227@qq.com

*Contributed equally

Key words: gastrointestinal stromal tumour, cell line, imatinib mesylate, resistance mechanism, GIST-T1
Flow cytometric cell cycle analysis indicated that the numbers of cells in the $G_{0} / G_{1}, S$ and $G_{2}$ phases increased following the induction treatment. Taken together, an IM-resistant GIST T1 cell line was successfully established, which opens novel avenues for individualized tumour chemotherapy.

\section{Introduction}

Imatinib mesylate (IM) is a targeted drug, which is specific for the treatment of gastrointestinal stromal tumours (GISTs) and is the standard first-line treatment for patients with unresectable primary GISTs or metastasized recurrent GISTs (1). IM can competitively inhibit the binding of ATP by binding to the ATP-binding site of the tyrosine kinase receptor, which inhibits a variety of tyrosine kinases, inhibits signal transduction, suppresses tumour growth, and can treat disease $(2,3)$.

Although numerous patients have benefitted from IM, certain patients are primarily resistant to IM, and the majority of patients exhibit drug resistance within 18-24 months, which leads to disease progression and patients even succumbing to mortality (4). To date, the mechanism underlying this resistance remains to be elucidated (5-9). The occurrence of drug resistance is the main reason for the failure of GIST treatment; therefore, investigations of the resistance mechanisms are currently a focus of investigations on GISTs. In the present study, the GIST-T1 cell line was used to establish a GIST-T1-resistant cell line (GIST-T1 IR) by intermittent dosing with increasing IM concentrations.

\section{Materials and methods}

Cell culture. GIST-T1 cells (IFO 50075; Human Science Research Resource Bank, Osaka, Japan) were cultured in RPMI 1640 (Gibco; Thermo Fisher Scientific, Inc., Waltham, MA, USA) medium containing $15 \%$ foetal bovine serum (TransGene Biotech, Beijing, China) and incubated at $37^{\circ} \mathrm{C}$ with $5 \% \mathrm{CO}_{2}$.

Induction of drug-resistant cells. To intermittently dose the cells with increasing IM concentrations, drug-containing medium was applied to the cells in the logarithmic growth phase. Following culturing for $48 \mathrm{~h}$, the medium was replaced 
with drug-free medium. The cells were then passaged until confluent. The administration of the specific IM concentration was continued until the cells grew normally. Subsequently, the concentration was increased and the above process was repeated to obtain a drug-resistant cell line through repeated induction. The CCK-8 assay results (Dojindo Molecular Technologies, Inc., Kumamoto, Japan) revealed that the half maximal inhibitory concentration $\left(\mathrm{IC}_{50}\right)$ was $10 \mu \mathrm{M}$. The drug administration for induction was initiated at a concentration of $2.5 \times 10^{4}$ of the $\mathrm{IC}_{50}(25 \mu \mathrm{M})$ followed by $37.5 \mu \mathrm{M}$ and a gradual increase to $50 \mu \mathrm{M}$.

Cellular morphological observation. Hematoxylin and eosin (H\&E) staining at $25^{\circ} \mathrm{C}$ for $3 \mathrm{~min}$, was performed on slides containing adherent cells for the morphological observation of the cells and to observe changes in the nuclei stained by Hematoxylin under an inverted microscope.

Determination of the cell growth curve and calculation of the doubling time. The two types of cells were digested with trypsin solution when they reached a confluence of $90 \%$ under a microscope and seeded into 24-well plates at a concentration of $5 \times 10^{4}$ cells $/ \mathrm{ml}$. The living cells were counted every $24 \mathrm{~h}$ for 5 days consecutively, and the results of three wells were averaged. The growth curve was prepared with the results of the daily cell counting. The majority of the cell proliferation was 'two division'. All data were fit into an exponential fitting model in SSPS 20.0 software (IBM SPSS, Armonk, NY, USA), according to the following formula: $\mathrm{Y}=\mathrm{ae}^{\mathrm{btln} 2}$, where $\mathrm{Y}$ is the number of cells, $t$ is the time of continuous culture, and a and $\mathrm{b}$ are curve constants. The cell doubling cycle was calculated using the following equation: $\mathrm{T}=\mathrm{t} \times \lg 2 / \lg \left(\mathrm{N}_{\mathrm{t}} / \mathrm{N}_{0}\right)$, where $\mathrm{T}$ is the population doubling time, $t$ is the time of continuous culture, $\mathrm{N}_{\mathrm{t}}$ is the final number of cells, and $\mathrm{N}_{0}$ is the initial number of cells. All time was measured in hours.

Cell scratch assay. The GIST-T1 and GIST-T1 IR cells were digested with trypsin for passage when they reached a confluence of $80-90 \%$ to obtain $70 \%$ confluence at $12 \mathrm{~h}$. An even scratch was introduced onto the bottom of the culture plate, which was followed by two PBS washes. The media were then replaced with complete medium containing $2 \%$ FBS and the drug at a concentration of 0 or $25 \mu \mathrm{M}$.

Determination of the cell cycle. The GIST-T1 and GIST-T1 IR cells in the logarithmic growth phase were digested with trypsin and centrifuged at $3,800 \times \mathrm{g}$ and $4^{\circ} \mathrm{C}$ for $3 \mathrm{~min}$. Ice-cold $75 \%$ ethanol was added, followed by incubation at $4^{\circ} \mathrm{C}$ overnight for fixation. Following centrifugation at $3,800 \times \mathrm{g}$ and $4^{\circ} \mathrm{C}$ for $3 \mathrm{~min}$, the supernatant was discarded. Following washing twice and resuspension in PBS, propidium iodide (PI) and RNase were added, and the cells were incubated at $4^{\circ} \mathrm{C}$ in the dark for $30 \mathrm{~min}$. Subsequently, the sample was centrifuged at 3,800 x g and $4^{\circ} \mathrm{C}$ for $3 \mathrm{~min}$ and the supernatant was discarded. Following washing once with PBS, the sample was transferred into a flow cytometry tube for detection on the instrument.

Determination of the $I_{50}$ for the two cell lines with the CCK-8 assay and calculation of the resistance index (RI). The GIST-T1 and GIST-T1 IR cells in the logarithmic growth phase were digested with trypsin and resuspended following centrifugation. The cell density was adjusted to 5,000 cells/100 $\mu \mathrm{l}$. A $100-\mu 1$ cell suspension was added to each well. When the cells were adherent, the GIST-T1 cell medium was replaced with fresh medium containing $2.5,10,15,20,25,30,40$ or $50 \mu \mathrm{M}$ IM. The GIST-TI IR cell medium was replaced with fresh medium containing $5,25,30,35,40,50,62.5$ or $75 \mu \mathrm{M}$ IM. Six duplicate wells were set up for each concentration, and all experiments included a drug-free control and a blank control. Following culture for $48 \mathrm{~h}$ under the same conditions, $10 \mu \mathrm{l}$ of the CCK- 8 reagent was added to each well. The absorbance was measured following incubation for $1 \mathrm{~h}$. The $\mathrm{IC}_{50}$ values were calculated using Graph Pad Prism 6 software (GraphPad Software, Inc., La Jolla, CA, USA). The dose response (variable slope) model in GraphPad Prism 6 software was used to fit the cell inhibition rate curve. The equation was as follows:

$$
\mathrm{Y}=\text { Bottom }+(\text { Top-Bottom }) /\left(1+10^{\wedge}\left(\left(\log \mathrm{IC}_{50}-\mathrm{X}\right)^{*} \text { HillSlope }\right)\right)
$$

$\mathrm{IC}_{50}$ is the concentration of agonist, which results in a response halfway between Bottom and Top. HillSlope describes the steepness of the family of curves. Top and Bottom are plateaus in the units of the $\mathrm{Y}$ axis.

Statistical analysis. All data were statistically analyzed using SPSS 20.0 software (IBM Corps., Chicago, IL, USA) and GraphPad Prism 6.0 (GraphPad Software, Inc.). The results are presented as the mean \pm standard deviation. Comparison of different groups was performed by one-way analysis of variance, followed by Tukey's post-hoc test for multiple comparisons. The dose response (variable slope) model in GraphPad Prism 6 software was used to fit the cell inhibition rate curve and the exponential fitting model in SSPS 20.0 software was used to fit the cell growth curve. $\mathrm{P}<0.05$ was considered to indicate a statistically significant difference.

\section{Results}

Establishment of the IM-resistant GIST-T1 IR cell line. The GIST-T1 cells were continuously cultured with media containing various concentrations of IM for $48 \mathrm{~h}$. The cells were observed and recorded at 24 and $48 \mathrm{~h}$. Based on the above preliminary experiment and considering a variety of factors, treatment with $25 \mu \mathrm{M}$ IM for $48 \mathrm{~h}$ was selected as the starting condition for the experiment. Following repeated treatments with stepwise increases in the drug concentration over 6 months, an IM-resistant GIST-T1 IR cell line was successfully induced. No significant change in RI was found following continuous cultured of the cells in the drug-free medium for 1 month. In addition, GIST-T1 IR cells thawed following cryopreservation remained drug-resistant without a statistically significant change in $\mathrm{IC}_{50}$ value, which suggested that the resistance of the GIST-T1 IR cells was stable. Therefore, these cells can be used as a model for studies investigating drug resistance.

Following induction with IM at 25 and $37.5 \mu \mathrm{M}$ concentrations, the concentration was gradually increased to $50 \mu \mathrm{M}$. When the cells became stable, an appropriate concentration gradient of the drug was determined in a preliminary experiment. The $\mathrm{IC}_{50}$ concentrations of the parental cells and the 

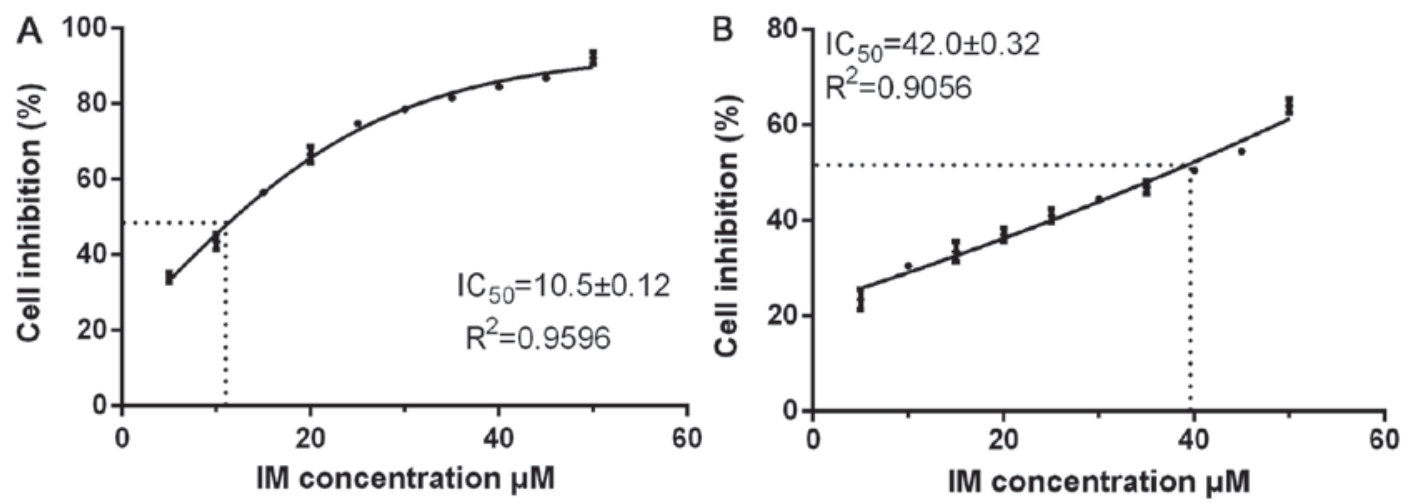

Figure 1. GIST-T1 and GIST-T1 IR cells. (A) GIST-T1 cell density was adjusted to 5, 000 cells/100 $\mu 1$, and the cells were cultured in 2.5, 10, 15, 20, 25, 30, 40 and $50 \mu \mathrm{M}$ IM. (B) GIST-TI IR cell medium contained 5, 25, 30, 35, 40, 50, 62.5 and $75 \mu \mathrm{M}$ IM, and the cells were cultured at the same cell density. GIST, gastrointestinal stromal tumour; IM, imatinib mesylate; IR, IM-resistant.
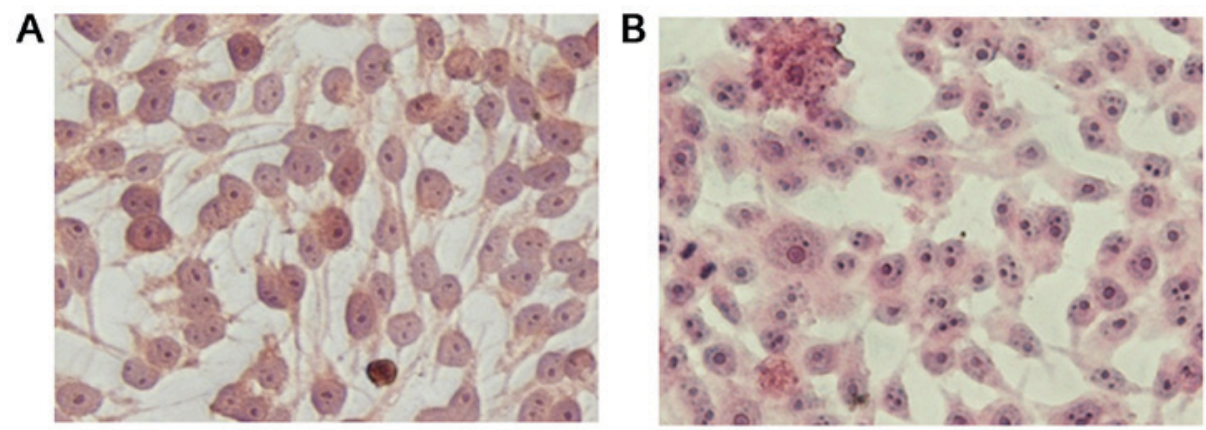

Figure 2. GIST-T1 and GIST-T1 IR hematoxylin and eosin staining (x400 magnification). (A) GIST-T1 cells were elongated, spindle-shaped, mononuclear or binuclear, and had a relatively large volume. (B) GIST-T1 IR cells had a smaller volume, darkly stained nuclei, were predominantly binuclear, triple-nuclear or multiple-nuclear, and had large nuclei. IR, imatinib mesylate-resistant; $\mathrm{IC}_{50}$, half maximal inhibitory concentration.

resistant cells were repeatedly detected to determine the drug resistance of the cells. As shown in Fig. 1A and B, the mean $\mathrm{IC}_{50}$ of the parental cells was $10.5 \pm 0.12 \mu \mathrm{M}\left(\mathrm{R}^{2}=0.9596\right.$, $\mathrm{P}<0.05)$, and the RI of the resistant cells was $42.0 \pm 0.32 \mu \mathrm{M}$, $\left(\mathrm{R}^{2}=0.9056, \mathrm{P}<0.05\right)$. The $\mathrm{RI}$ was $\sim 4.0$, and, in the range of $1-5$, the resistance of the cells was in the low range. The $\mathrm{R}^{2}$ values of the curve of cell inhibition rate close to 1 , indicating that the above models simulated the cell inhibition curve well.

Differences in the biological behaviours of the GIST-T1 and GIST-TI IR cells. Slides containing adherent parental and resistant cells were observed under an inverted microscope following H\&E staining. The results, as shown in Fig. 2A and $\mathrm{B}$, indicated that the GIST-T1 cells were elongated, spindle-shaped, mononuclear or binuclear, and had a relatively large volume. The GIST-T1 IR cells had a smaller volume with darkly stained nuclei, were predominantly binuclear, triple-nuclear or multiple-nuclear, and had large nuclei.

The cell growth curves are shown in Fig. 3. The average proliferation times calculated by consecutive counting based on the equation for the GIST-T1 and GIST-T1 IR cells were $26.59 \pm 1.34 \mathrm{~h}\left(\mathrm{R}^{2}=0.9280, \mathrm{P}<0.05\right)$ and $33.63 \pm 2.82 \mathrm{~h}$ $\left(\mathrm{R}^{2}=0.9883, \mathrm{P}<0.05\right)$, respectively, and this difference represented a significant extension of $\sim 7.04 \mathrm{~h}$ for the GIST-T1 IR cells $(\mathrm{P}<0.05)$. The $\mathrm{R}^{2}$ values of the two cell growth curves indicated that the above models simulated the cell growth well. The two cell types did not exhibit a significant difference over 1-2 days. Cell proliferation was in a relatively static stage. Subsequently, the difference in the cell proliferation rate gradually increased and was marked within 3-5 days. After 5 days, the average total numbers of cells observed to be actively proliferating were $1.1 \times 10^{6}$ for the GIST-T1 cells and only $6 \times 10^{5}$ for the resistant cells. Additionally, the resistant cells were found to be more dependent on the highly nutritious medium, compared with the untreated cells in this experiment. A graph of the cells in the different phases of the cell cycle is shown in Fig. 4. The distributions of the GIST-T1 and GIST-T1 IR cells in the different phases of the cell cycle were 16.46 and $20.72 \%$ in the $S$ phase, 33.45 and $39.88 \%$ in the $\mathrm{G}_{0} / \mathrm{G}_{1}$ phase, and 28.48 and $19.01 \%$ in the $\mathrm{G}_{2} / \mathrm{M}$ phase, respectively. The GIST-T1 IR distribution was increased by $\sim 4.26 \%$ in the $\mathrm{S}$ phase and $\sim 6.43 \%$ in the G0/G1 phase, and was decreased by $\sim 9.47 \%$ in the $\mathrm{G}_{2} / \mathrm{M}$ phase. The differences were considered significant at $\mathrm{P}<0.05$.

The effects of $2 \%$ FBS medium and a drug concentration of $25 \mu \mathrm{M}$ on the parental and resistant cells were investigated using the scratch assay. The results of the scratch assay are shown in Fig. 5. The GIST-T1 cells and GIST-T1 IR cells migrated towards the centre in the absence of the drug. The migration ability of the GIST-T1 cells was greater than that of the GIST-T1 IR cells under the same conditions in the absence of drug treatment. When the drug was added at the concentration of $25 \mu \mathrm{M}$, no significant migration of the GIST-T1 cells was observed, which demonstrated significant inhibition. By contrast, the GIST-T1 IR cells migrated and did not exhibit a significant difference, compared with the cells in the group 
with no drug. Therefore, IM significantly inhibited the migration of the GIST-T1 cells, compared with the cells under the same condition with no drug, whereas its effect on the GIST-T1 IR cells was weaker.

\section{Discussion}

The main methods used to establish drug-resistant cells include the large dose impact method, drug concentration increasing method, and large dose impact with concentration increasing method (5). In the present study, the drug concentration increasing method was applied to induce the GIST-T1 cell line. Compared with a study on GIST-882 cell line induction, the GIST-T1 line is difficult to induce due to its attribute of an IM-sensitive subtype (6). The method used to induce tumour cell resistance in the present study was in line with the process of acquired resistance of tumour cells, and can more accurately simulate the biological changes following tumour resistance. The drug-resistant cell lines were subjected to DNA and RNA sequencing, with no new gene mutations found on DNA sequencing. The results of RNA-sequencing showed that a number of drug-related genes were abnormally expressed. Similar studies have found the gene expression profiles change in IM-resistant cell line, including the upregulation of genes associated with tumor-related genes MYC and Frizzled class receptor 10, which may be involved in tumorigenesis (7). The expression of Erb-B2 receptor tyrosine kinase 4 was found to be the most marked, which was similar to KIT and was activated as an alternative pathway following IM treatment, resulting in tumor resistance to IM $(7,8)$. In addition, the expression of a large number of genes associated with drug metabolism have been found, including aldehyde oxidase 1, cytochrome P450 family 3 (CYP3)A4, CYP3A5 and UDP glucuronosyltransferase family $1 \mathrm{~A} 1$, which are also involved in the metabolism of IM, leading to functional resistance. The majority of the downregulated genes belong predominantly to cell junction communication, tumor inhibition and complement defense (8). Examinations on the function of these drug resistance-associated genes are ongoing, therefore, data are not available in the present study.

Primary IM resistance is the wild-type GIST, which is without mutations in the c-kit gene. Even in the KIT mutant type of GIST, the different mutant reaction sites to IM are not the same. A clinical study identified that the effective rate of exon 11 mutation subtype GIST was $87.3 \%$ and exon 9 mutation subtype was $47.8 \%$, while the exon 13 and exon 17 mutation subtypes were invalid for the treatment with IM as IM could not effectively block the activation of mutations in the intracellular segment of KIT due to mutations of exon 13 and 17 (9). The exon 13 and exon 17 mutation subtype GIST are IM sensitive in vitro, it is possible that the metabolism of IM in vivo affects the IM affinity of exon 13 and exon 17 mutation subtypes GIST. However, their clinical efficacy remains to be elucidated and resistance mechanisms need to be further studied. The present clinical study found that not all wild-type KIT and GISTs were resistant to IM, although certain types showed positive results. The present study found a mutation in the PDGFRA gene in these patients. The PDGFRA gene mutations were mainly located in exon 12 and exon 18; for the exon 12 mutation, it was

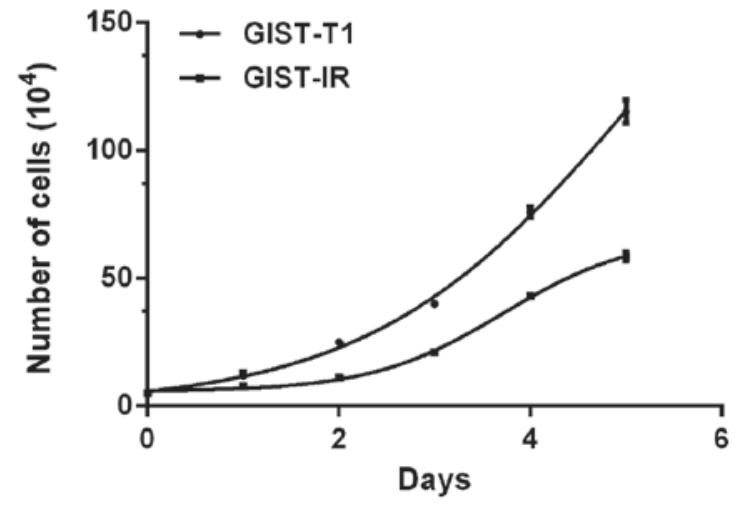

Figure 3. GIST-T1 and GIST-T1 IR cell growth curves. The GIST-T1 and GIST-T1 IR cells were seeded into 24-well plates at a concentration of $5 \times 10^{4}$ cells $/ \mathrm{ml}$ and counted every $24 \mathrm{~h}$ for 5 days consecutively. IR, imatinib mesylate-resistant.

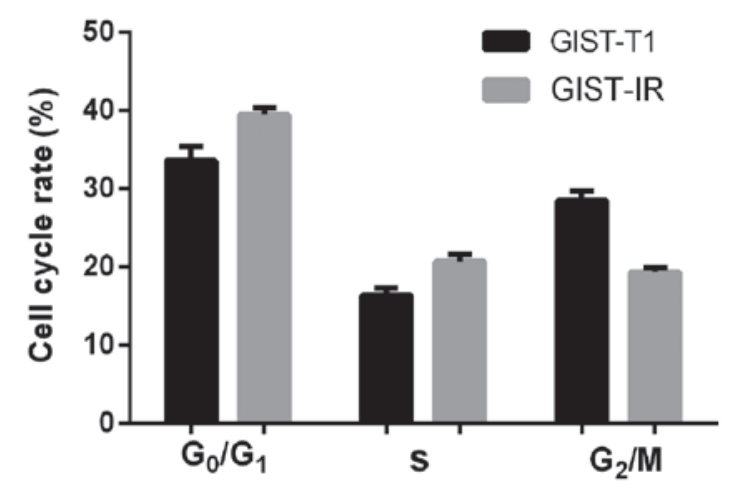

Figure 4. GIST-T1 and GIST-T1 IR cell cycle. The GIST-T1 and GIST-T1 IR cell cycles were measured by flow cytometry. IR, imatinib mesylate-resistant.

experimentally confirmed that the subtype was IM-sensitive in vitro. In clinical studies, it has also been shown that the drug of this subtype has certain curative effects; exon 18 mutations of $\mathrm{D} 842 \mathrm{~V}$ mutant strains resistant to IM subtype in the presence of other mutant strains are susceptible to the drug. The mechanism of secondary resistance to IM remains to be fully elucidated, although no gene overlapping with primary drug resistance has been found in gene detection of secondary drug-resistant cell lines (10). This suggests that the primary drug resistance mechanism of GIST is different from secondary acquired resistance, and it is necessary to confirm the above difference using in vitro drug resistance assessments. The construction of in vitro drug resistance model is critical.

IM as a first-line target drug use to treat GIST and is the most widely recognized drug for the treatment of solid tumours. Resistance is common following IM treatment for GIST and is currently a major problem for GIST treatment. Studies have demonstrated that $\sim 50 \%$ of patients with GIST who are susceptible to IM experience secondary resistance to IM treatment after 2-2.5 years, which seriously affects the prognoses of the patients $(11,12)$. The mechanism of IM resistance has become one of the focuses of current investigations in tumour molecular biology. The in vitro establishment of a drug-resistant tumour cell line is an effective method in cancer research and has been generally applied for the investigation 

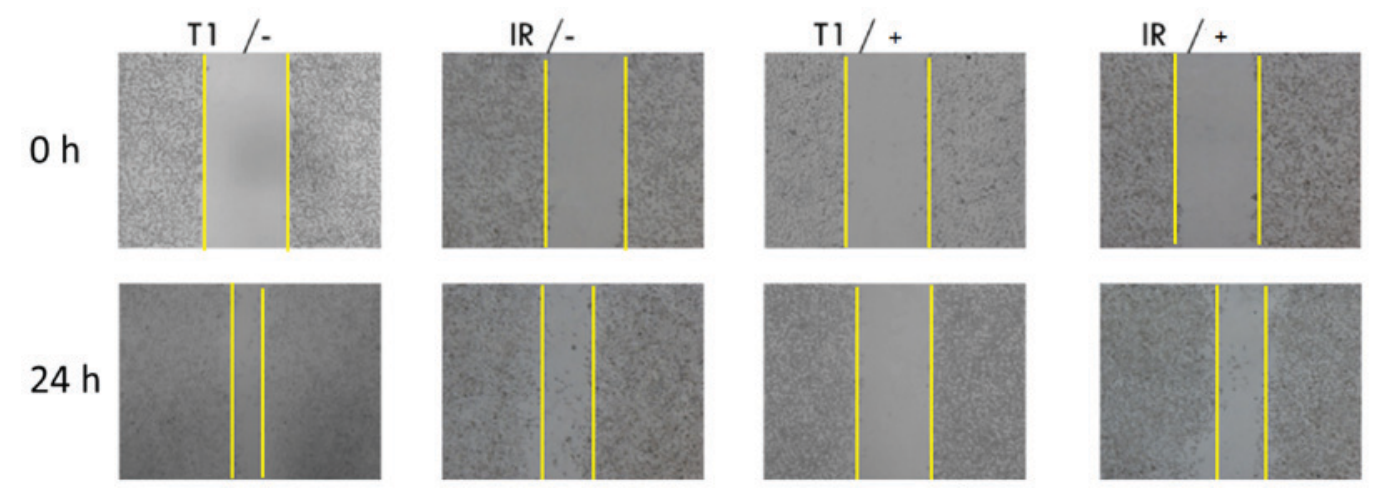

Figure 5. GIST-T1 and GIST-T1 IR cell scratch assay. The GIST-T1 and GIST-T1 IR cells were treated with IM at a concentration of 0 (-) or 25 (+) $\mu \mathrm{M}$. Migration was observed following incubation for $24 \mathrm{~h}$. IM, imatinib mesylate; IR, IM-resistant; T1, non-resistant.

of drug resistance in China and other countries. This method has the advantages of a relatively simple experimental procedure, readily controllable conditions and relatively low costs. Therefore, the induction of an IM-resistant GIST-T1 cell line is a simple, direct and effective approach for the investigation of GIST resistance and its reversal.

The present study used GIST-T1 cells as the study subject. An IM-resistant GIST-T1 cell line, termed GIST-T1 IR, was successfully established via repeated induction with gradually increasing doses over 6 months. The comparison of the biological characteristics of the GIST-T1 and GIST-T1 IR cells revealed that the growth of the cells with induced resistance was marginally slower, the nucleus-to-cytoplasm ratio was increased, and the cells were predominantly multinucleated with more cellular particulate matter when their tolerance to IM was increased. The changes in the cell cycle detected using flow cytometry revealed that more GIST-T1 IR cells were in the $S$ and $G_{0} / G_{1}$ phases, and fewer cells were in the $G_{2} / M$ phase, compared with the GIST-T1 cells. These results indicated that the mitosis of the drug-resistant cells was reduced, and the rate of DNA synthesis was reduced. Therefore, more cells were retained in the $\mathrm{G}_{0}$ phase to reduce energy consumption due to reproduction. Subsequently, this saved energy was utilized for resistance to the drug. The migration assay revealed that the resistant cells continuously migrated in the presence of the drug, and exhibited an enhanced ability to migrate with an increased degree of malignancy.

A change to the morphologies of resistant cells is one of the indicators of resistance. The comparison of the GIST-T1 cells and the drug-resistant GIST-T1 IR cells in the present study revealed that the drug-resistant cells were smaller and less smooth, with an irregular cell contour and ill-defined boundary. The number of nuclei was increased with the abnormal morphology, in addition to and increased nuclear-cytoplasmic ratio and mitotic appearance. The growth curve of the cells revealed that the cell proliferation rate was reduced and their proliferation was significantly weaker, compared with that of the untreated cells. The cell doubling time was $33.63 \mathrm{~h}$, which was significantly longer than the $26.59 \mathrm{~h}$ observed for the parental cells.

In the present study, the $\mathrm{G}_{0} / \mathrm{G}_{1}$ ratio of the GIST-T1 IR cells was increased by $\sim 6.43 \%$, compared with that of the GIST-T1 cells ( $\mathrm{G}_{0}$ is the resting phase of the cells). One explanation is that substantial energy was consumed for drug resistance; therefore, the energy supply available for the other life activities of the cells was reduced, and the syntheses of proteins and DNA required for mitosis was reduced, which resulted in the retention of cells in the resting phase. However, the regulation of cell cycle proteins may be important in the changes in the cell cycle during the process of resistance. This finding is consistent with the results of other studies that have investigated the cell cycle in drug-resistant cell lines $(13,14)$. The number of cells in the $\mathrm{S}$ phase was increased by $\sim 4.26 \%$, whereas the number of cells in the $\mathrm{G}_{2} / \mathrm{M}$ phases was decreased by $\sim 9.47 \%$. The increase in the number of cells in the $\mathrm{S}$ phase suggested an increase in DNA synthesis, whereas the decrease in the number of cells in the $\mathrm{G}_{2} / \mathrm{M}$ phases indicated a reduction of cells in the mitotic stage and that cell division may be arrested in the $\mathrm{G}_{2}$ phase.

The results of the scratch assay revealed that the drug-resistant cells continued to migrate in the presence of the drug with an enhanced migration capacity and an increased degree of malignancy. When the effectiveness of IM on GIST is weakened, and the patient becomes resistant to the drug, subsequent metastasis of GIST to the liver, abdomen and other organs may occur. According to the results of the present study, IM can significantly inhibit the migration of tumour cells with no drug resistance, which can suppress tumour metastasis (15). However, the capacity of IM to inhibit metastasis was weakened in the GIST-T1 IR cells, and the metastasis of tumour cells still occurred at certain IM concentrations. Based on these results, it was hypothesized that the migration capacity of the tumour cells was regained in the presence of the drug following the development of drug resistance, which may be one important reason for metastasis in patients (16). One possible explanation for the lower strength of this migration compared with that in the parental cells is that the energy and components in the drug-resistant cells were redistributed to retain its resistance capacity. Therefore, components, including proteins, required for migration were decreased, which resulted in weaker migration ability, compared with the ability prior to the development of drug resistance (17). Further investigations are required to elucidate the specific mechanism.

Zhen et al (18) investigated the susceptibilities of two different cell lines (GIST-T1 and GIST-882) to IM and found a marked difference (18). The proliferation of GIST-T1 cells was inhibited by IM at the concentration of $0.1 \mu \mathrm{M}$, whereas the proliferation of GIST-882 cells was not inhibited 
until the IM concentration reached $1.0 \mu \mathrm{M}$. In the study of Nakatani et al (19), The GIST-T1 cells were more sensitive to IM, and the induction of their resistance was lower with a lower level of induction. These findings were consistent with the results of the present study. The mutations found in exon 11 of GIST-T1 were the same as those detected in the majority of clinical patients with stromal tumours, which reflects a good IM response and significant clinical effect. However, mild or serious drug resistance is elicited by extending the period of drug treatment, which is also consistent with the clinical situation.

In conclusion, an IM-resistant GIST cell model (GIST-T1 IR) was successfully established in the present study based on the clinical characteristics of the chemotherapeutic drug. The GIST-T1 cells acquired resistance to IM, but also showed changes in cell morphology, proliferation, migration and cell cycle, suggesting there is a complex mechanism in the process of tumour resistance. Therefore, the construction of drug-resistant cell lines can provide a solid foundation for further investigations of the mechanism of acquired resistance and provide a reliable example of targeting drug-induced resistance. The model exhibited the basic biological properties of resistant cells, and provides an elementary experimental model for further investigations of IM resistance mechanisms and the screening of effective drugs for the treatment of GIST.

\section{Acknowledgements}

This study was funded by the National Natural Science Foundation of China (grant no. 81172380). The authors would like to thank Dr Yangyu Feng (Life Sciences, Fuzhou University, Fujian, China) for advice and critical manuscript reading.

\section{Competing interests}

The authors declare that they have no competing interests.

\section{References}

1. Milhem M and Deutsch JM: Imatinib dosing in gastrointestinal stromal tumors (GISTs): When, how much, and how long. Curr Clin Pharmacol 10: 311-320, 2015.

2. Lee JH, Kim Y, Choi JW and Kim YS: Correlation of imatinib resistance with the mutational status of KIT and PDGFRA genes in gastrointestinal stromal tumors: A meta-analysis. J Gastrointestin Liver Dis 22: 413-418, 2013

3. Yan L, Zou L, Zhao W, Wang Y, Liu B, Yao H and Yu H: Clinicopathological significance of c-KIT mutation in gastrointestinal stromal tumors: A systematic review and meta-analysis. Sci Rep 5: 13718, 2015.

4. Yang J, Ikezoe T, Nishioka C, Takezaki Y, Hanazaki K, Taguchi T and Yokoyama A: Long-term exposure of gastrointestinal stroma tumor cells to sunitinib induces epigenetic silencing of the PTEN gene. Int J Cancer 130: 959-966, 2012.
5. Serranogarcia C, Heinrich MC, Zhu M, Raut CP, Eilers G, Ravegnini G, Demetri GD, Bauer S, Fletcher JA and George S: In vitro and in vivo activity of regorafenib (REGO) in drug-resistant gastrointestinal stromal tumours (GIST). Vet Radiol Ultrasound 54: 365-372, 2013.

6. Corless CL, Barnett CM and Heinrich MC: Gastrointestinal stromal tumours: Origin and molecular oncology. Nat Rev Cancer 11: 865-878, 2011.

7. Prakash S, Sarran L, Socci N, DeMatteo RP, Eisenstat J, Greco AM, Maki RG, Wexler LH, LaQuaglia MP, Besmer P and Antonescu CR: Gastrointestinal stromal tumors in children and young adults: A clinicopathologic, molecular, and genomic study of 15 cases and review of the literature. J Pediatr Hematol Oncol 27: 179-187, 2005.

8. Frolov A, Chahwan S, Ochs M, Arnoletti JP, Pan ZZ, Favorova O, Fletcher J, von Mehren M, Eisenberg B and Godwin AK: Response markers and the molecular mechanisms of action of Gleevec in gastrointestinal stromal tumors. Mol Cancer Ther 2: 699-709, 2003.

9. Chou TY, Chiu CH, Li LH, Hsiao CY, Tzen CY, Chang KT, Chen YM, Perng RP, Tsai SF and Tsai CM: Mutation in the tyrosine kinase domain of epidermal growth factor receptor is a predictive and prognostic factor for gefitinib treatment in patients with non-small cell lung cancer. Clin Cancer Res 11: 3750-3757, 2005.

10. Takahashi T, Elzawahry A, Mimaki S, Furukawa E, Nakatsuka R, Nakamura H, Nishigaki T, Serada S, Naka T, Hirota S, et al: Genomic and transcriptomic analysis of imatinib resistance in gastrointestinal stromal tumors. Genes Chromosomes Cancer 56: 303-313, 2017.

11. Cao G, Li J, Shen L and Zhu X: Transcatheter arterial chemoembolization for gastrointestinal stromal tumors with liver metastases. World J Gastroenterol 18: 6134-6140, 2012.

12. Hsueh YS, Lin CL, Chiang NJ, Yen CC, Li CF, Shan YS, Ko CH, Shih NY, Wang LM, Chen TS and Chen LT: Selecting tyrosine kinase inhibitors for gastrointestinal stromal tumor with secondary KIT activation-loop domain mutations. PLoS One 8: e65762, 2013

13. Xu J and Cao H: Molecular mechanism and therapeutic strategy for resistance to tyrosine kinase inhibitors in targeted treatment of gastrointestinal stromal tumors. Zhonghua Wei Chang Wai Ke Za Zhi 16: 288-291, 2013 (In Chinese).

14. Buleje SJ, Yábar BA, Guevara-Fujita M and Fujita R: Molecular and genetic features of gastrointestinal stromal tumors (GIST). Rev Gastroenterol Peru 32: 394-399, 2012.

15. Patel S: Exploring novel therapeutic targets in GIST: Focus on the PI3K/Akt/mTOR pathway. Curr Oncol Rep 15: 386-395, 2013.

16. Heinrich MC, Griffith D, McKinley A, Patterson J, Presnell A, Ramachandran A and Debiec-Rychter M: Crenolanib inhibits the drug-resistant PDGFRA D842V mutation associated with imatinib-resistant gastrointestinal stromal tumors. Clin Cancer Res 18: 4375-4384, 2012.

17. Zhu HL, Liu T, Meng WT and Jia YQ: Establishment of an imatinib resistance cell line K562R and its resistant principia. Sichuan Da Xue Xue Bao Yi Xue Ban 38: 22-26, 2007 (In Chinese).

18. Zhen S, Wang H, Leng HJ, Tong WJ, Tao D and Pan YL: Establishment of an imatinib resistance cell line and Identification of biological characteristics. Chin J Experimental Surg 29: 550-551, 2012 (In Chinese)

19. Nakatani H, Araki K, Jin T, Kobayashi M, Sugimoto T, Akimori T, Namikawa T, Okamoto K, Nakano T, Okabayashi T, et al: STI571 (Glivec) induces cell death in the gastrointestinal stromal tumor cell line, GIST-T1, via endoplasmic reticulum stress response. Int J Mol Med 17: 893-897, 2006.

c) (7) $\ominus$ This work is licensed under a Creative Commons Attribution-NonCommercial-NoDerivatives 4.0 International (CC BY-NC-ND 4.0) License. 